\title{
TRACKING MOVEMENT OF BODY-MOUNTED MARKERS THROUGH OPAQUE ORTHOTICS
}

Petra Aradi ${ }^{1}$, Gábor István Horváth ${ }^{1}$, András Czmerk ${ }^{1}$, Tamás Terebessy ${ }^{2}$, Péter Tamás ${ }^{1}$

${ }^{1}$ Budapest Univeristy of Technology and Economics, Faculty of Mechanical Engineering, Department of

Mechatronics, Optics and Engineering Informatics

${ }^{2}$ Semmelweis University, Department of Orthopedics

aradi.petra.bme@gmail.com

\section{Abstract}

It is very important in marker-based motion capture systems that cameras can follow markers attached to anatomical points without any obstruction. Based on marker positions the computer program can reconstruct movement of the body's biomechanical model by calculating typical distances, angles and trajectories.

Analysis becomes complicated when the person wears a rigid and opaque orthotic device (e.g. a plastic corset), through which cameras can not see markers attached to the body surface.

We had to find a marker attachment method, so that infrared motion capture cameras can follow movement of markers attached to certain anatomical points on scoliosis patients wearing their corsets, when analyzing the effects of corsets on posture and movement.

Infrared cameras in the Gait Analysis Laboratory of Semmelweis University's Department of Orthopedics can not ,see through” polyethylene corsets. Markers have to be attached directly to the body surface, because a corset is a rigid body, therefore it moves differently, than the human body under it. Multiple considerations have to be made, when choosing markers. There is limited room between body and corset, markers can not affect wearer's movement and can not cause discomfort. The chosen method uses magnets attached to the skin with medical tape. Size and properties of magnets were verified by simulation. Infrared cameras can follow the painted magnet counterparts on the outer surface of the corset.

Magnets attached to the corset's outer surface follow the movement of magnets taped to the body, so cameras can follow movement of anatomical points under the opaque corset, without the need of providing direct visibility by destructing corset material. Holes larger than the size of markers would be required, to avoid the shading effects of the corset resulting from body-corset and body-camera distance and angle.

According to measurement results of patients with and without corsets, magnetic markers sufficiently follow the body's movement under the corset. In the meantime with markers attached immovably to the corset's outer surface, movement of the corset as a rigid body can be tracked, as well.

The presented method allows further detailed investigation, concerning the movement altering effects of not just corsets, but other orthotics, too.

Keywords: biomechatronics, spinal disorders, magnetic sensors, corset, motion capture system 


\section{Introduction}

The number of juvenile scoliosis patients is increasing worldwide. Part of conservative treatment is the use of rigid orthotic devices, called corsets ${ }^{1}$. It is important to analyze the effects of corsets, which can be done with gait analysis ${ }^{2}$, since the spine plays a central role in posture and movement.

The infrared marker based motion capture system of Semmelweis University's Department of Orthopedics was used to test various sensor configurations. Markers are attached to the person's body in specific anatomical points, which the motion capture system uses for computer reconstruction of movement. Markers to be used by persons wearing a corset have to:

- be small enough to fit between corset and body surface

- $\quad$ weigh little, so that movement is not notably affected

- be detectable through the corset's polyethylene material

- $\quad$ be as small as possible, so that they can be considered point wise

- be easily attachable and eliminate skin motion

- $\quad$ be safe to wear and be of no health risk

A number of trials showed, that widely used polyethylene corsets do not allow infrared cameras to ,see through" them, that is "regular" markers attached to the body under the corset are invisible for cameras. Various methods were tested, and finally magnetically attached markers provided satisfactory results. Magnetic attachment properties were tested and simulated, than evaluated with the motion capture system.

The basis of work presented in this paper was carried out in the frame of Gerincőr (SpineGuard) Project, as a thesis ${ }^{3}$ project submitted as partial fulfillment of BSc in Mechatronic Engineering degree, by the second author, under the supervision of the other authors.

\section{Methods}

\section{Motion capture system setup}

Motion capture systems use markers attached to anatomically relevant body points. The above mentioned motion capture system uses six static infrared cameras. Markers that these cameras can detect are attached to certain body points with medical adhesive as shown in Figure 1. The test person walks along a predefined path, meanwhile cameras record position of markers. After that the software processes the acquired data and shows reconstructed 3D movement on screen. Wavy lines on Figure 1 show the position of markers during walking. 


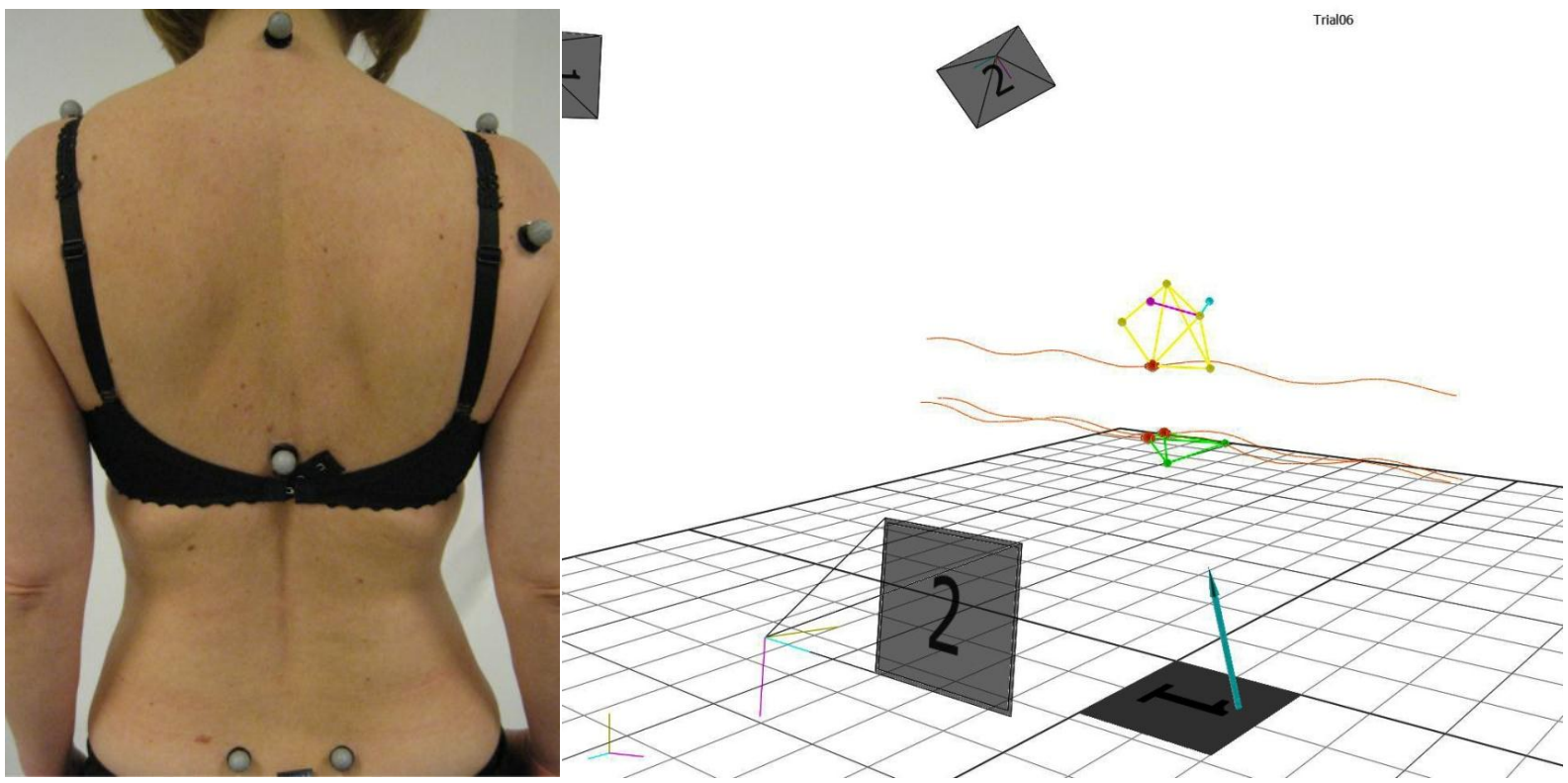

Figure 1. Infrared markers attached to skin and their recorded movement

The effects of wearing a corset appear not just in static posture, but during movement, as well. An important aspect would be the possibility to compare movement with and without corset. Infrared markers under the corsets are not just invisible for cameras, but as one can see in Figure 1 , in most cases are too big to fit under the corset. The conclusion is that this measurement system can not be used for testing patients wearing an opaque corset.

The very first idea, namely attaching the markers normally used on skin, onto the corset, above the predetermined points is of no practical use. It can surely show the corset's movement, however the important aspect would be to follow the body's movement under the rigid corset.

\section{Cutting holes on the corset}

One possibility is to cut holes on the corset where markers need to be placed. It is not optimal, because holes destruct corset material, and they might even be large enough to endanger corset's structural integrity. Holes do have to be of larger diameter than markers, to allow the minimal movement of markers together with skin movement and to avoid the shading effects of the corset resulting from variable body-corset and body-camera distance and angle, especially during movement.

\section{Infrared LEDs as markers}

Another option is the use of infrared LEDs attached to the skin as markers. This option has quite a number of drawbacks. However LEDS and the necessary electronics including batteries can be small enough (well under a square centimeter), so size is not a problem, but it requires quite high power to light through the corset, which means a large amount of dissipated heat, making it uncomfortable to wear. 
Still, there were experiments made with LED panels, after the most appropriate components were selected and simulated with SPEOS program (Figure 2).

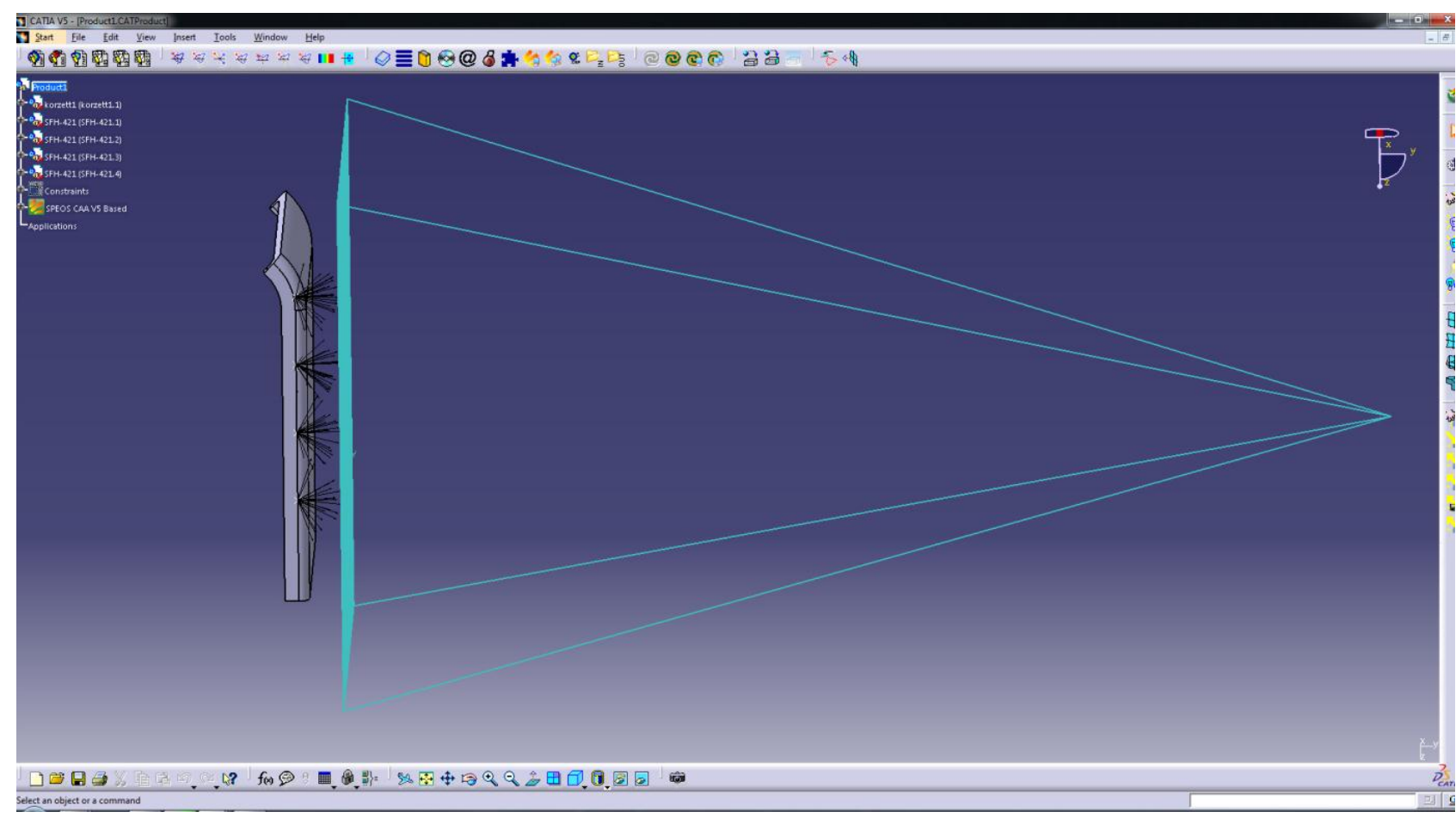

Figure 2. SPEOS light intensity simulation of chosen infrared LEDs placed on the body under the corset

After obtaining the convincing simulation results, an experimental setup was built and tested with the motion capture system. LEDs were mounted under a corset and placed into the laboratory. The results showed that the selected setup is not applicable, because cameras could not detect infrared light from LEDs hidden behind the corset. Without the opaque corset, in frared LED markers were perfectly detectable with the cameras, even during movement.

The contradictive results of simulation and measurement might be traced back to the too simplified corset model geometry, which does not take into account light scattering on the real corset surface.

\section{Magnets as bridges between skin and corset}

The idea of magnetic motion capture systems is already present in literature ${ }^{4}$, but because of the limited range of the mentioned system and the need to use the available infrared motion capture system, another approach had to be evaluated.

The basic idea is the use of permanent magnet pairs, one of which is attached to the skin in desired anatomical points, while the other magnet is on the outer side of the corset. The latter 
magnet is covered with special dye that makes it visible for infrared cameras. When the marker magnet on the body moves, its pair also changes position, what cameras can follow.

Magnets have to satisfy the six requirements specified in Introduction. So, magnet pairs have to be lightweight, small in size, but have to provide sufficient cohesion. The chosen magnets are made of rare earth elements, and are usually nickel plated. Nickel is a metal that often causes allergic skin reactions, so magnets can not be covered with nickel to avoid health risks.

Cylindrical magnets were chosen, and their geometric model and material properties were introduced in finite element simulation (Figure 3), to optimize magnet dimensions that provide appropriate cohesion.

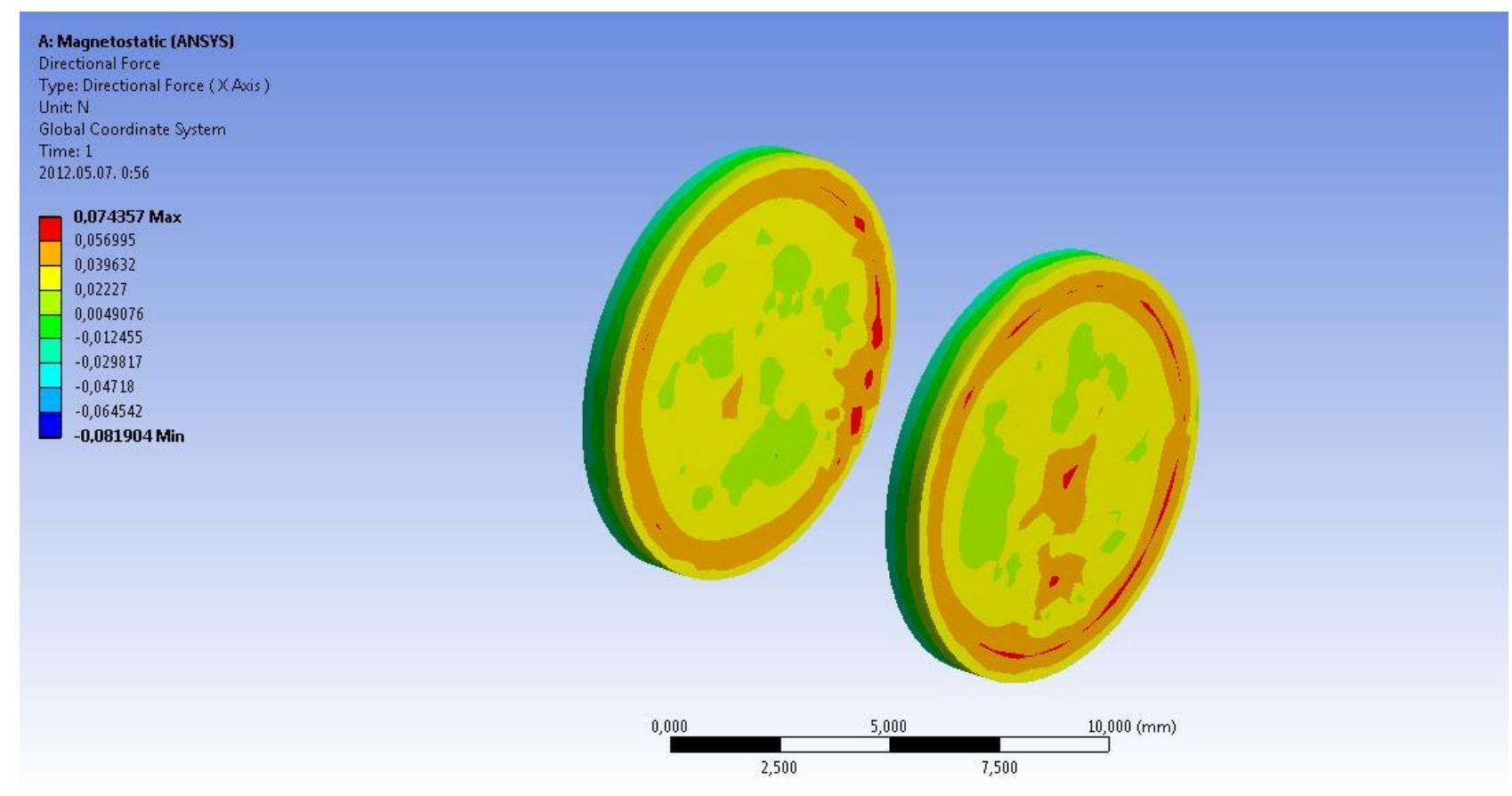

Figure 3. Finite element simulation of magnet pair

Further tests were carried out with parametric models to determine relationship among magnet size (radius and height), distance between magnets and forces. One of the results among three of four parameters, magnet radius, (half the) distance and force between magnets is shown in Figure 4. Similar graphs were obtained for the other two available parameter combinations.

Constraints had to be defined for magnet height, because $4 \mathrm{~mm}$ high magnets can be placed on the skin without touching the corset from inside. The other limit $(1 \mathrm{~mm})$ is the height of commercially available disk shaped magnets.

A parameter sensitivity diagram (Figure 5) was also obtained from the program, showing that force between magnets is mostly affected by distance (távolság), the negative sign meaning inverse proportionality. The second affecting parameter is magnet height (vastagság), where the positive sign represents direct proportionality. 


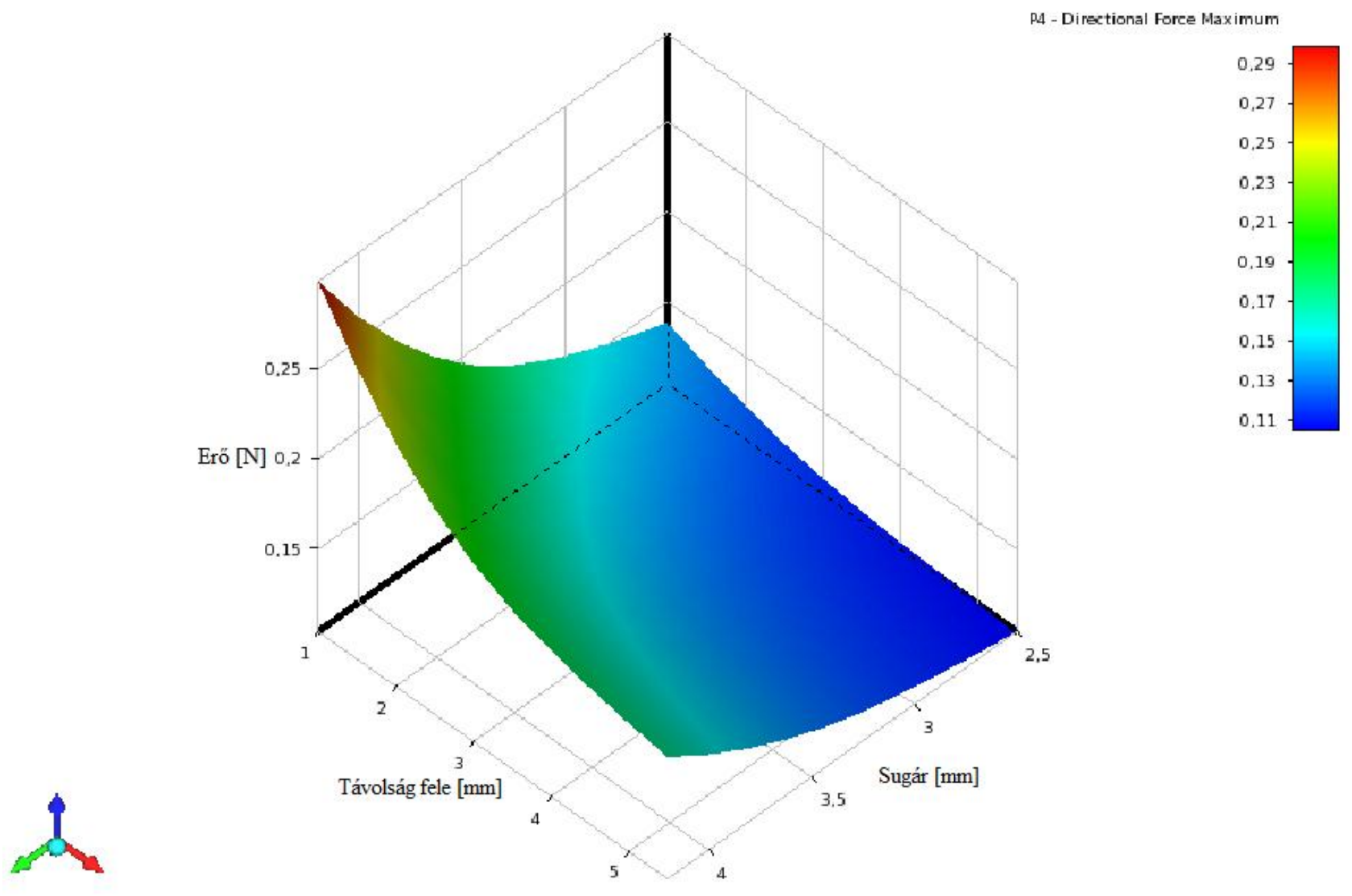

Figure 4. Results of parametric tests among magnet radial size (sugár), distance (távolság) and force (erô)

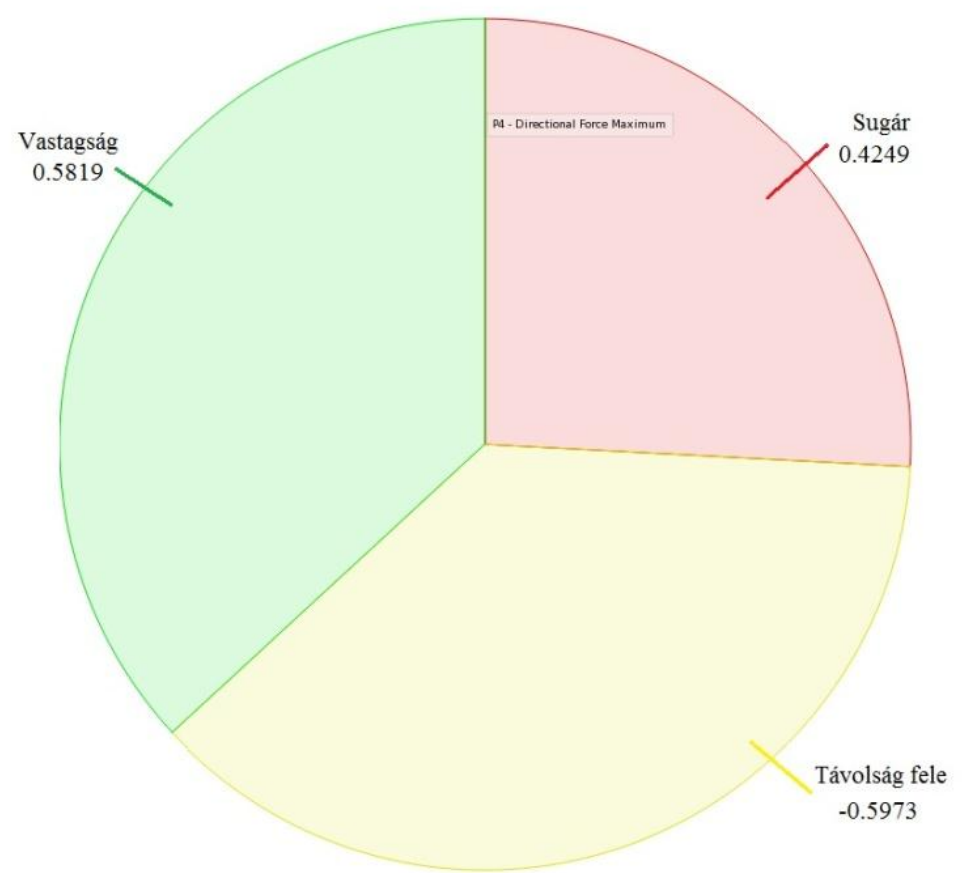

Figure 5. Parameter sensitivity diagram of force, effects of height (vastagság), radius (sugár), (half the) distance (távolság) 
After simulation results were evaluated and magnets were obtained, tensile strength tests were performed to create a mathematical model of force as a function of distance (Figure 6).
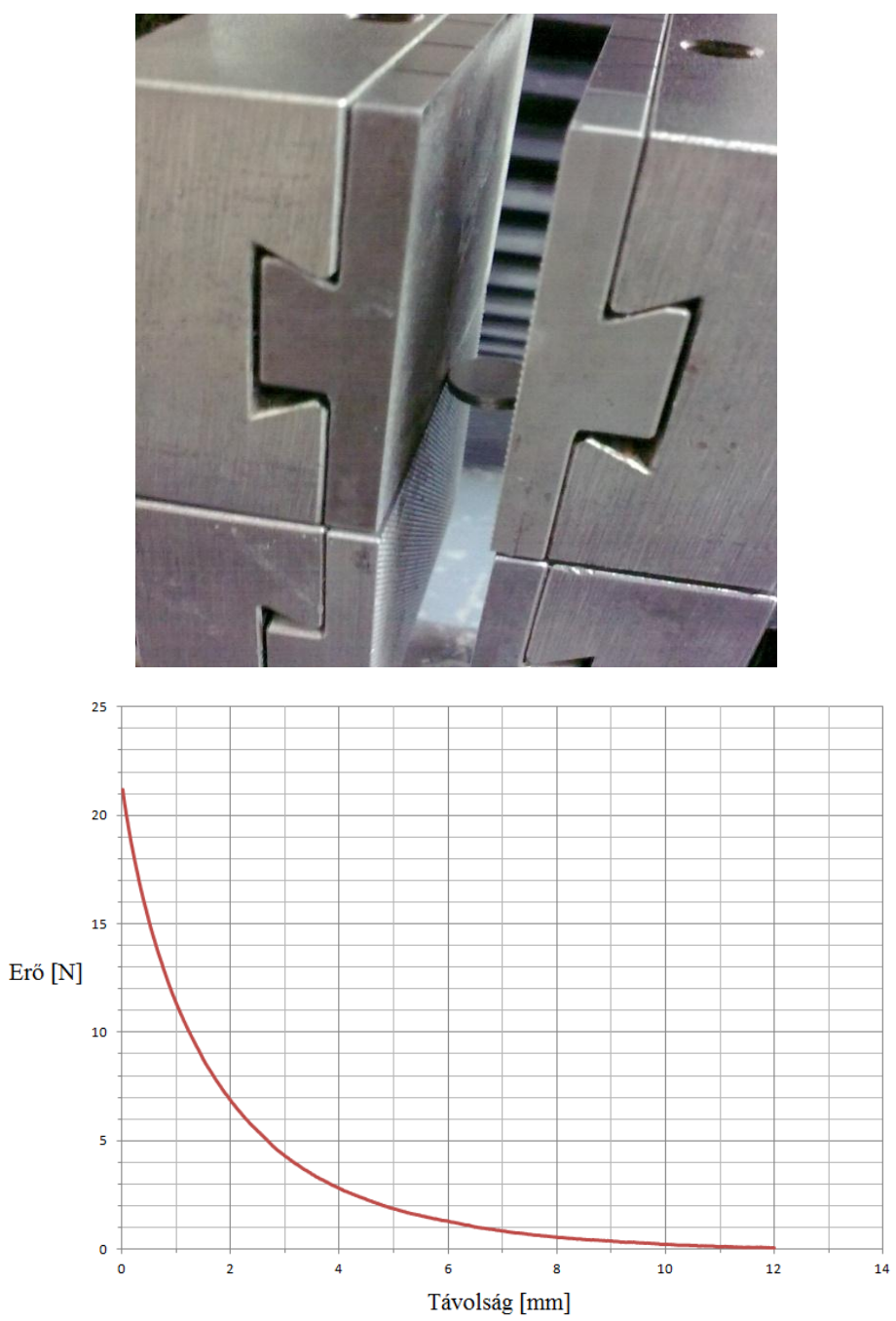

Figure 6. Magnet in tensile strength tester and measurement result, distance (távolság)-force (erô) diagram

Tensile strength tests confirmed simulation results; however simulation results have a great numerical deviation from measurement results, caused probably by model simplifications.

Another test was performed to determine dead band that is when a magnet moves just a little, the other magnet might not start to move (Figure 7). 


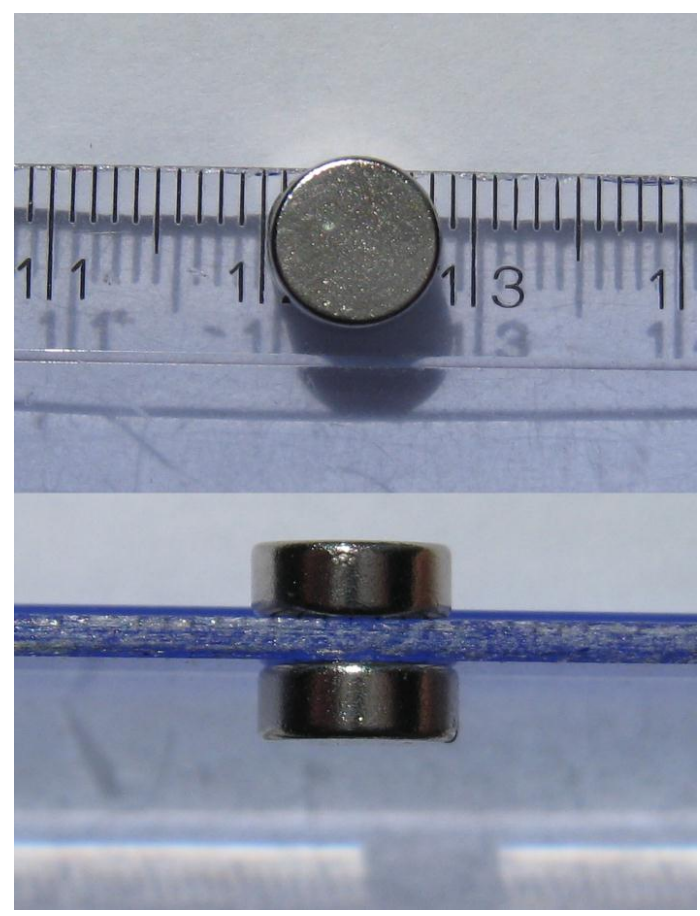

Figure 7. Determination of dead band

Magnet pair interaction also had to examined, to avoid the unwanted interaction of two magnets on the outside of the corset. It can happen, when the predefined anatomical points are so near to one another that their outside magnets start to draw each other, instead of reacting to the movement of their respective pairs on the body surface. A minimal distance was determined for magnets on the body surface, as well as one for the outer ones.

When the first corset tests were performed with three magnet pairs, the anatomical points used were lumbar spinous processes, another problem surfaced. At two of the three points, the distance between the magnet on the skin and the corset was too big, so various sized spacers had to be created. Spacers have to be lightweight, and have to be magnetically connectable on both ends.

\section{Results}

After tests without corset and with the original markers (Figure 1), magnets (and where needed spacers) were attached to the body and their counterparts were put to the corset outside. Results shown in Figure 8 justify the presented magnetic marker motion capture principle.

Spacers introduce some uncertainty in measurement, so further analysis is necessary to eliminate it. 


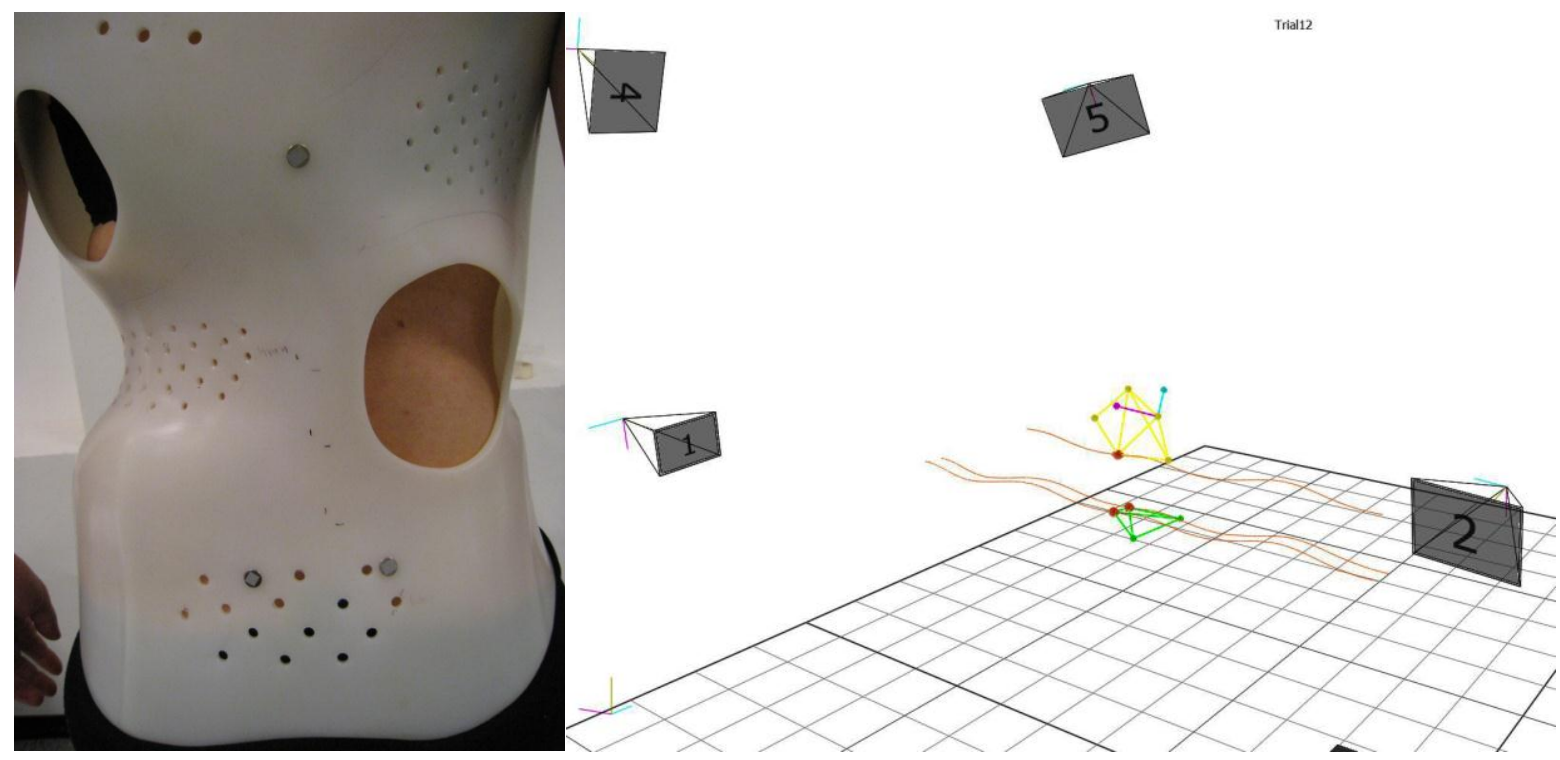

Figure 8. Magnetic marker pairs and their recorded movement

\section{Conclusion}

A method with such inexpensive accessories deserves further tests and investigations, which are under way, to validate this promising measurement principle. Other types of plastic orthotic devices can be tested with the method, too. Different tests can be designed and examination protocols written, so that not just the effects of corsets on walking could be tested.

\section{REFERENCES}

1. Szendrői M (editor). Ortopédia. Budapest: Semmelweis Kiadó, 2009. ISBN: 9789639879492

2. Kocsis L, Kiss RM, Illyés Á. Mozgásszervek biomechanikája. Budapest: Terc Kiadó, 2007. ISBN: 978 9639535640

3. Horváth GI. Motion capture through non-transparent material, BSc thesis in Mechatronics (major in Biomechatronics), Budapest university of Technology and Economics, Department of Mechatronics, Optics and Engineering Informatics, 2012.

4. Hashi S, Toyoda M, Yabukami S, Ishiyama K, Okazaki Y, Arai KI, Kanetaka H. Wireless magnetic motion capture system using multiple LC resonant magnetic markers with high accuracy. Sensors and Actuators A 142 - 2008: 520-27.

The authors would like to thank National Development Agency (NDA) of Hungarian Government for its support since this study has been carried out commonly as part of project GERINCO2 TECH_08-A1/2-2008-0121. 